\title{
Visual Homograph: A Novel Basic Visual Element
}

\author{
Liyan Zhang ${ }^{1,3}, \mathrm{Ke} \mathrm{Gao}^{2}$ \\ ${ }^{1}$ College of Computer Science and Technology, Nanjing University of Aeronautics and \\ Astronautics \\ 2 Institute of Computing Technology, Chinese Academy of Science \\ 3 Collaborative Innovation Center of Novel Software Technology and Industrialization
}

\begin{abstract}
The bag-of-visual-words (BoW) model has been broadly applied to represent images in a variety of image retrieval applications. Ideal visual word vocabulary should obtain two important properties, completeness and distinctiveness (i.e., containing all the similar image patches and eliminating different ones accurately). However, the traditional definition of basic visual elements tend to lack in either generality or particularity, which will lead to the incoherence problem and further ruin the image retrieval performance. To address this issue, in this article, we propose a novel basic visual element called Visual Homograph - groups of local patches which have indivisible descriptors but differ in origins. To generate Visual Homograph based vocabulary, we also propose a novel similarity metric named Integrated Perceptual Similarity which systematically combines center-based and boundary-based measures. Experimental results on standard datasets demonstrate the superiority of Visual Homograph based method over state-of-the-art visual vocabulary generation approaches, in terms of alleviating incoherence problem, and enhancing retrieval performance in practical applications.
\end{abstract}

Keywords: Image retrieval, Integrated Perceptual Similarity, Visual Homographs

Email address: zhangly84@126.com (Liyan Zhang ${ }^{1,3}$ )

Preprint submitted to Journal of Neurocomputing

March 30, 2016

(C) 2016. This manuscript version is made available under the Elsevier user license http://www.elsevier.com/open-access/userlicense/1.0/ 


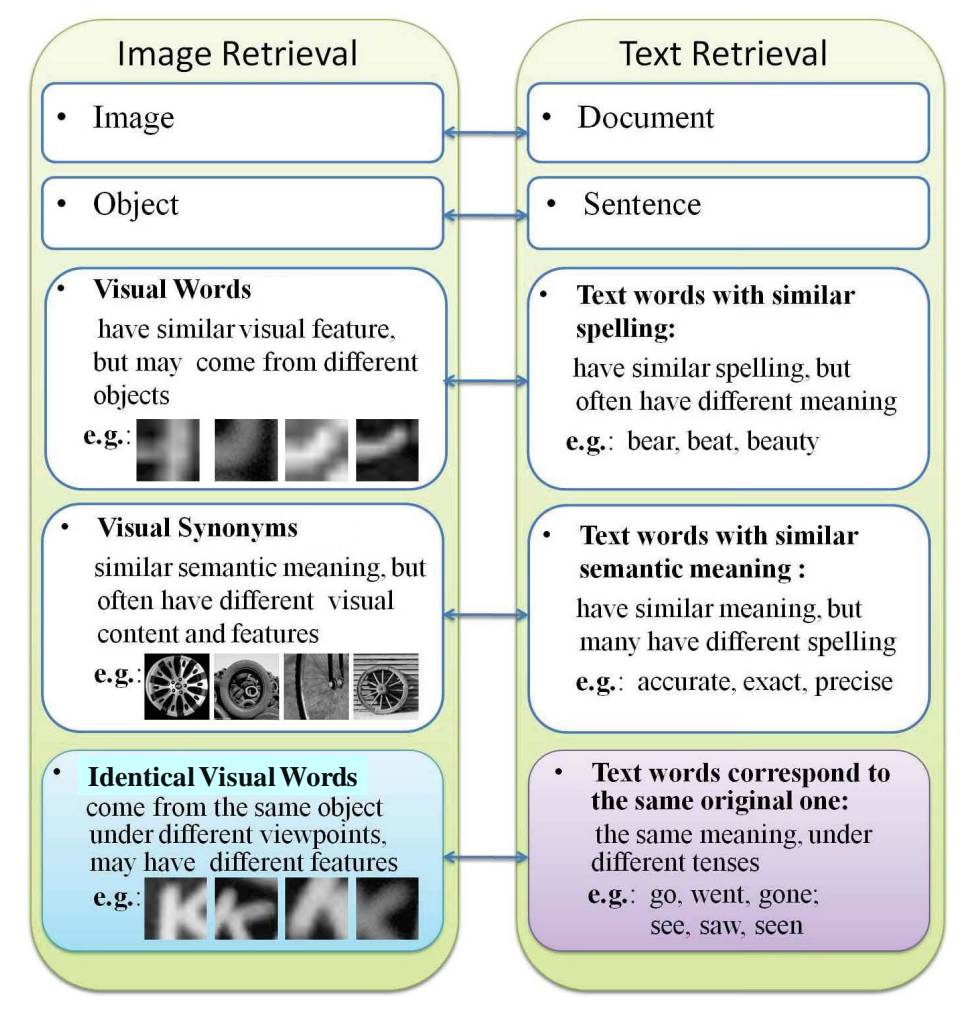

Figure 1: An analogy between image and text retrieval.

\section{Introduction}

Inspired by text retrieval, bag-of-visual-words(BoW) model has been successfully applied in a variety of image retrieval applications [1, 2, 3, 4]. Visual words are regarded as clusters of similar local patches, and generated based on a

5 simple assumption - the descriptors of similar patches will be close to each other in some appropriate metric. Based on the BoW description, many algorithms in text retrieval such as inverted file and context analysis can be directly applied to large-scale image retrieval applications [5, 6, 7].

However, in contrast to words in text retrieval, such intrinsic entities do not exist in the visual domain. Therefore, the generation of visual words/elements from image patches is an essential component, and has been extensively explored in the prior literature. As illustrated in Figure 1, the existing basic visual ele- 


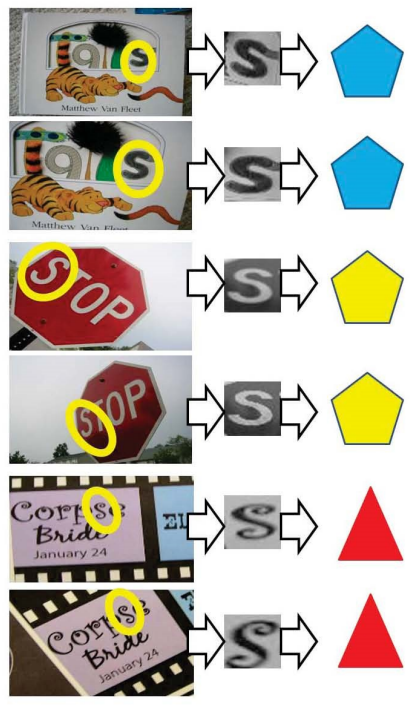

Other local patches

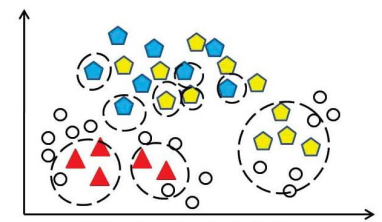

(a) Visual Words

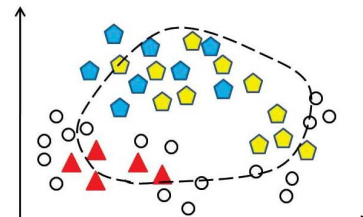

(b) Visual Synonyms

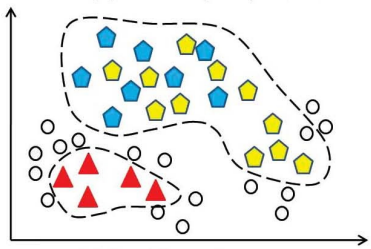

(c) Our Visual Homographs

Figure 2: Interpretation and some instances of different basic visual elements.

ments can be summarized as: traditional visual words [2], visual synonyms [8] and identical visual words [9]. These visual elements respectively corresponds to three types of patch similarity metrics, Euclidean similarity [2, 4], semantic similarity [10, 11] and origin similarity [9, 8, Traditional visual words are created by clustering image patches in unsupervised ways based on local feature Euclidean distance. Traditional visual words are the most common and popular visual elements, however, the performance is not satisfied since Euclidean distance between descriptors is a good indicator of patch similarity only in certain range. Visual synonyms leverage supervised methods to learn semantic similarities for particular object recognition. However, the patch similarities are often defined towards particular object recognition, and thus lack extensibility-only suitable for classification tasks within limited number of object categories. Identical visual words refer to the patches which come from the same object/origin under different viewpoints. The performance is unsatisfied because of the large variation of local patches and descriptors.

Due to the limitation of existing basic visual elements (the definition of patch 

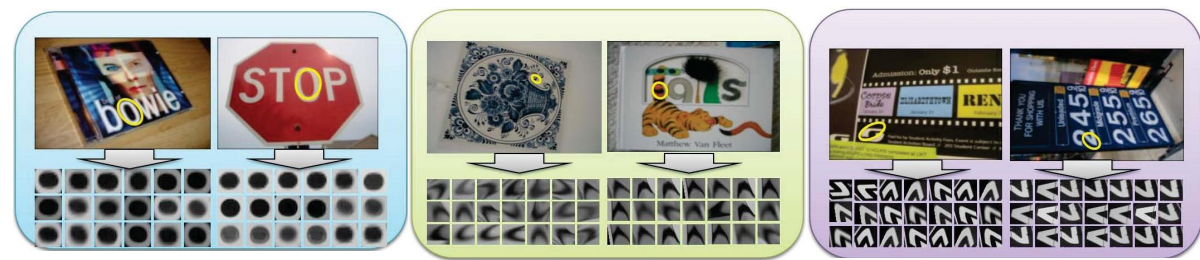

Figure 3: Instances of Visual Homographs which have indivisible descriptors but differ in origins.

similarity tend to be either too general or too specific), the performance of visual patch clustering is not ideal. As illustrated in Figure 2(a)(b), the results from traditional visual words tend to be over-partitioning (similar patches are assigned into different groups), while results from visual synonyms turn to be under-partitioning (noise/errors are included in clusters). This phenomenon is called "Incoherence Problem" 8].

To address this issue, in this article, we aim to explore the appropriate definition of basic visual elements in order to construct the complete and distinctive visual vocabulary (that is to say, all of the similar patches should be clustered together and different ones should be eliminated accurately). Inspired by text homograph (group of words that have the same spelling but differ in meanings),

40 in this article, we propose a novel definition of basic visual elements called Visual Homographs, which denotes groups of image patches with similar appearance (with indivisible descriptors) but may come from different objects, see Figure 3 . These patches with similar appearance should not be separated, although they come from different objects/origins. Separating them coercively will lead to 45 the over-partitioning problem, and also result in a very huge visual vocabulary. Moreover, to establish the relationship between these over-partitioned words using exhaustively search in this huge vocabulary is too expensive. Therefore, Visual Homograph based vocabulary can significantly alleviate the incoherence problem.

To generate Visual Homograph based vocabulary, we also present a novel similarity metric called Integrated Perceptual Similarity which appropriately integrates center-based measure and boundary-based measure to generate local 
patch similarities. Experiments on standard datasets demonstrate that the proposed visual element-Visual Homograph can effectively alleviate the incoherence problem, and achieve better image retrieval performance, compared with conventional visual elements. We summarize the main contribution of this article as follows.

- Propose a novel definition of basic visual elements called Visual Homographs, to effectively alleviate the incoherence problem.

- Present Integrated Perceptual Similarity which appropriately integrates center-based measure and boundary-based measure to group the relevant visual patches.

- Describe the efficient approach to assign new patch to Visual Homograph based vocabulary.

- Conduct experiments on standard dataset to demonstrate the effectiveness and efficiency of the proposed method.

The rest of our work is organized as follows. We start by introducing conventional basic visual elements in Section 2. And then in Section 3, we present the proposed Integrated Perceptual Similarity, and the algorithm to generate and assign Visual Homographs. The proposed method is empirically evaluated in Section 4. Finally, we conclude in Section 5 by highlighting the key points of our work.

\section{Related Work}

In this section, we briefly introduce the three types of conventional visual elements (traditional visual words [2, visual synonyms [8] and identical visual words [9]), as well as the corresponding three kinds of patch similarity metrics.

Traditional visual words are created by clustering local descriptors of image patches in unsupervised ways using Euclidean distance, such as k-means and 
hierarchical clustering [2, 3]. Each cluster is taken as a visual word like Figure 2(a). A new descriptor is assigned to its nearest word, according to the Euclidean distance between the descriptor and cluster centers. Although visual word has been very popular in recent years, it is often proven not as effective as the textual words due to the incoherence problem [4, 8]. The reason might be that the Euclidean distance between descriptors is a good indicator of patch similarity only in certain range [9], thus the accuracy of patch classification will be seriously affected by clustering number, initial centers and noise data.

Since only relying on Euclidean distance is too general to classify patches accurately, researchers have developed many supervised methods to learn semantic similarities for particular object recognition [10, 11, 12, 13. By assigning semantic class labels such as "cars" and "bikes" to local descriptors, the semantic similarities between descriptors are learned with Extremely Randomized Clustering Forests [10], and patches sharing the same semantic label are supposed to be clustered into the same visual words. Based on a universal vocabulary, particular class vocabularies such as cat and dog vocabularies are obtained through the adaptation of the universal vocabulary using class-specific data [11. These methods provide "middle -level" representations which help to bridge the semantic gap between the low-level features and the high-level concepts to be categorized. However, the patch similarities are often defined toward particular object recognition, thus lack extensibility and are only suitable for classification tasks within limited number of object categories. Moreover, due to the semantic gap, the incoherence problem especially "under-partitioning problem" is hard to avoid, that is to say, there are many patches belonging to different semantic categories existing in the same patch groups, as shown in Figure 2(b).

Recently, using a fine partitioned vocabulary to avoid the under-partitioning problem has been popular 9 , 8. Because only patches extracted from identical objects (the same origin) are considered to be similar, a very large vocabulary including several million words is constructed (one or two orders of magnitude larger than commonly used). Furthermore, although the probabilistic relationships between words are learned and stored additionally in [9, the 

descriptors.

More advanced methods have been proposed to optimize the vocabulary, such as soft assignment and Hamming embedding [4, 5, 14. Some prior work [15, [16, 17, 18, conduct a comprehensive study on the representation choices of BoW, including vocabulary size, weighting scheme, stop word removal, feature selection, spatial information, and visual bi-gram. However, since the basic definitions of patch similarity are not improved in essence, there is no guarantee that the patch classification of those methods can be complete and distinctive at the same time.

Therefore, it is a fundamental issue to define the patch similarity and construct a meaningful visual vocabulary. Only with a proper definition of patch similarity, can we find a proper way to generate complete and distinctive basic visual elements based on existing local feature extraction methods. Thus in this paper, we propose the novel definition of visual element - visual homograph, which denotes groups of image patches with similar appearance (with indivisible descriptors) but may come from different objects. In addition, we also propose a novel similarity metric called Integrated Perceptual Similarity to generate VH based vocabulary.

\section{Visual Homograph}

130

In this section, we mainly discuss the proposed basic visual element - Visual Homograph, which refers to groups of image patches with similar appearance (with indivisible descriptors) but may come from different objects. To generate Visual Homograph based vocabulary, we first introduce the novel similarity metric called Integrated Perceptual Similarity, and then discuss the generation and assignment for visual homograph based vocabulary.

\subsection{Integrated Perceptual Similarity}

The key issue in exploring a meaningful visual vocabulary is the definition of patch similarities/relevance. Based on the obtained relevance score, a given 

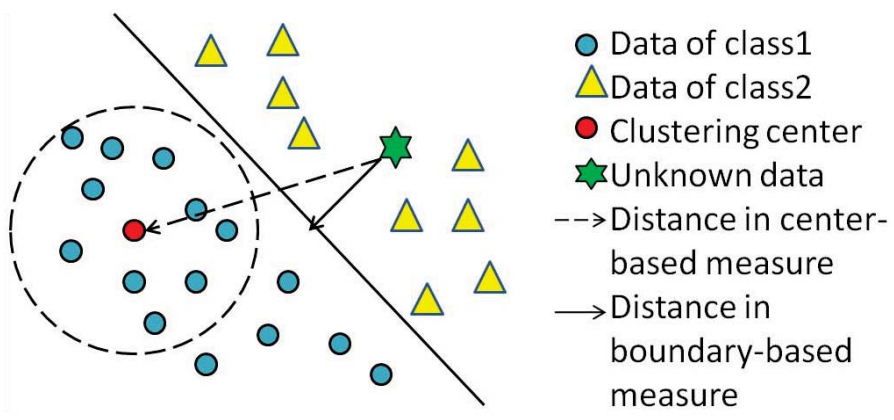

Figure 4: Two types of similarity calculation methods.

patch can be regarded as relevant or non-relevant to a particular visual group. In our case, according to the definition of visual homograph, we define the "relevant patches" as the patches with similar appearance but may come from different objects.

Generally, the existing relevance calculation methods can be broadly categorized into two categories: center-based measure and boundary-based measure, as illustrated in Figure 4. The traditional visual words and the identical visual words are often generated with center-based measure, while visual synonyms with similar semantic are obtained using boundary-based measure. The former is easy to calculate thus support fast search in huge database using indexes such as k-d tree or LSH [19, but it is a good indicator of patch similarity only in certain range 9 . The latter has better generalization by exploring the potential association between descriptions, but heavily rely on the choice of training data.

To generate and assign Visual Homographs, we propose "Integrated Perceptual Similarity" which systematically integrate the aforementioned two measures. As illustrated in Figure 5 a coarse-partitioning vocabulary is first calculated using center-based measure, such as k-means. Within each cluster, begin with patches sharing the same origins, every set of relative patches are found out using boundary-based measure based on similarity propagation. Such groups of patches are regarded Visual Homographs in this paper. Given a new patch, it will be assigned to the most similar Visual Homograph based on both 

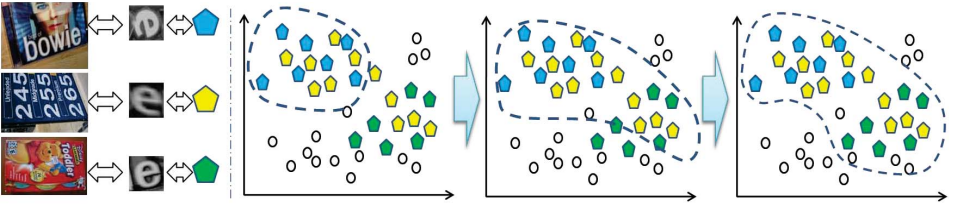

Figure 5: Visual Homographs generation based on similarity propagation.

160

Let $x_{1}, x_{2}, \ldots, x_{l}$ be relative patches belonging to a basic group X. Using a kernel map which transforms the training descriptors to another space, one- 
class SVM separates the positive data from the origin, by solving the following quadratic programming problem:

$$
\min \frac{1}{2}\|w\|^{2}+\frac{1}{v l} \sum_{i=1}^{l} \xi_{i}-\rho
$$

Subject to

$$
\left(w \cdot \Phi\left(x_{i}\right)\right) \geq \rho-\xi_{i}, \quad i=1,2, \ldots, l, \quad \xi_{i} \geq 0
$$

Where $l$ denotes the number of relative patches, and $\Phi\left(x_{i}\right)$ is the kernel map function. More details can be found in 22]. The algorithm for Visual Homographs generation is summarized in algorithm 1, and the process of similarity propagation is illustrated in Figure 4.

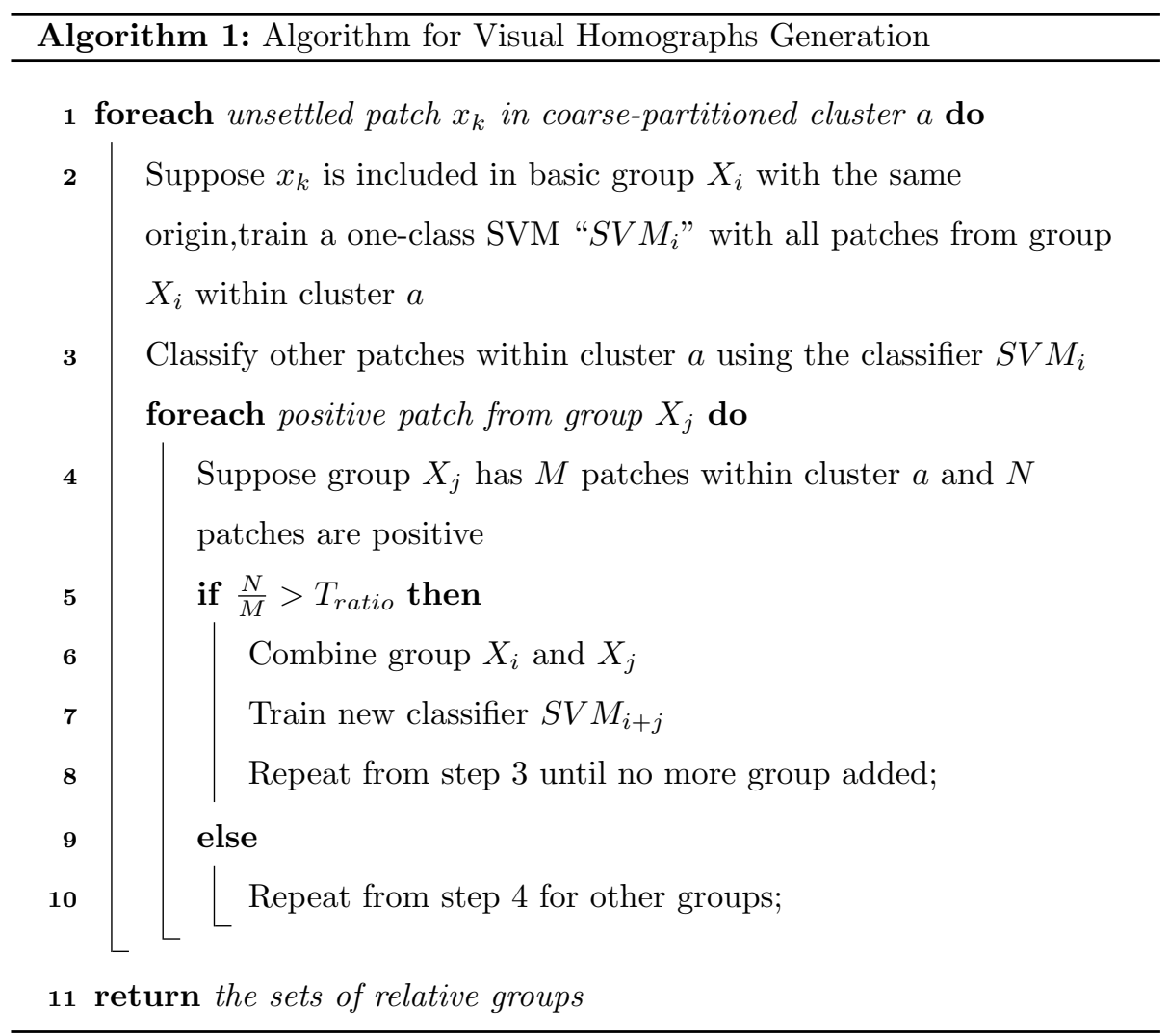


In this way, growing from a small and over-partitioned group of patches, an indivisible and complete set of relative patches can be obtained. And these sets of patches are called Visual Homographs in this paper.

\subsection{Visual Homographs Assignment}

195 assign a new patch to the most similar Visual Homographs effectively and efficiently is also very important for practical applications.

Aforementioned, Visual Homographs has been generated by combining two similarity metrics. Among them k-means is used to obtain a coarse-partitioning

\section{Experiments}

The goal of this work is to explore the novel basic elements to construct a visual vocabulary which are more complete and distinctive, in order to support a wide range of visual based applications. To evaluate the performance of the proposed "Visual Homograph" element, we conduct a series of experiments on several standard datasets, including the Ukbench dataset [3], INRIA holidays dataset [5] and internet partial-duplicate image database (IPD) [23]. The Ukbench dataset contains 10200 images totally, involving 2550 image categories; 
tances. The INRIA holidays dataset contains 500 image groups, each of which represents a distinct scene or object. The IPD dataset consists of 10 image collections which has 200 partial-duplicates in each, and 8,000 non-duplicate web images. Thus it contains totally 10,000 images, including 2000 images with 10 collections and 8000 non-duplicate images.

To extract local patches and descriptions, MSER detector and SIFT descriptor 24] are leveraged, since they can achieved the best repeatability and accuracy [24]. Although many modified versions are reported to obtain better performance in some particular tests, they are not suitable for a wide range of applications.

In the experiments, we mainly focus on three aspects: (a) The effectiveness of Visual Homographs in alleviating the incoherence problem. (b) Retrieval accuracy in practical applications. (c) The memory and time cost of Visual Homographs generation and assignment.

\subsection{Alleviation of Incoherence Problem}

In this subsection, experiments are conducted to demonstrate the effectiveness of the proposed Visual Homographs in alleviating the incoherence problem. To optimize the local similarity measures of patch similarity, a large training set is required. Enough instances for each object should be provided for training. Here, we use the Ukbench dataset [3] as the training set, which consists of 10200 images in 2550 image categories. To obtain more instances with different viewpoints and distances for each category, we leverage the expansion methods to generate more relative images. For each category, we generate 41 synthetic images. These synthetic images can be utilized for patch similarity learning. erated, which will be leveraged throughout the later experiments. Figure 3 demonstrated the examples of visual homographs generated in our experiments.

We compare the performance of Visual Homographs (VH) with several thestate-of-art vocabulary generation methods, including traditional visual words 


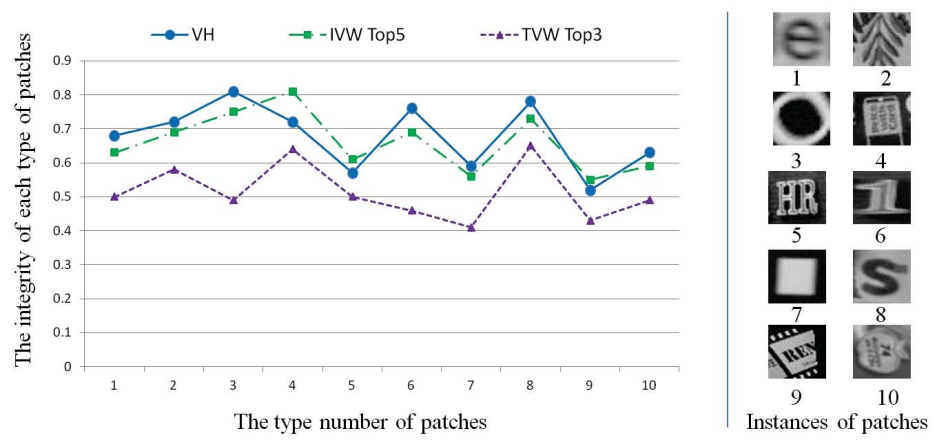

Figure 6: Integrity comparisons between three methods.

${ }_{250}$ (TVW) 4 and the identical visual words (IVW) 9]. In the experiments, 3 nearest TVWs are taken into account as suggested in paper [4] (TVW Top3), and 5 alternative words of IVW are utilized based on paper 9 (IVW Top 5 , the performance improves slowly when the number is larger than 5).

To measure the completeness and distinctiveness of the visual vocabulary, 255 we choose integrity as the evaluation metric. It refers to the ratio of corrected patches to all the clustered patches, as to each type of visual patches, denoted as,

$$
\text { integrity }=\frac{\sharp \text { corrected patches }}{\sharp \text { total patches }}
$$

In our experiments, to compute integrity, we select 10 types of patches randomly from different objects in the Ukbench dataset, and manually record the relative patches as ground truth. Note that total count of relative patches in this dataset is larger than 4 , since the original images are expanded to a large set of synthetic images.

Figure 6 illustrates the comparison between our Visual Homograph (VH) method with traditional visual words (TVW) and the identical visual words (IVW). As illustrated in Figure 6, the integrity of our VH method is comparable with IVW and much better than TVW. Besides, TVW is generated leveraging unsupervised clustering, which will lead to more noise and error patches. This comparison demonstrates the effectiveness of our VH method in alleviating 

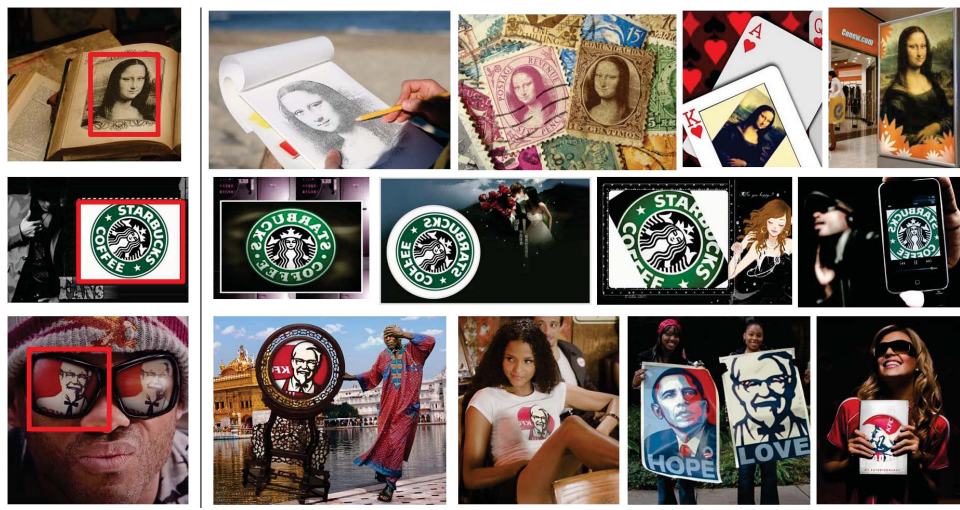

Figure 7: Some instances for NDD results using Visual Homographs (The leftmost is the query image).

incoherence problem.

Compared with IVW, the advantage of our approach is that we leverage the Integrated Perceptual Similarity to group image patches with similar appearance which might come from different objects. IVW only groups patches extracted from identical objects (the same origin), which will lead to a very large vocabulary and over-partitioning problem. Our approach can significantly alleviate the incoherence problem.

\subsection{Performance in Near-Duplicate image Detection}

To test the performance of our approach in practical application, we apply the VH based visual vocabulary to the task of Near-Duplicate image Detection (NDD). The notion of near-duplicate images simply refers to the images which share the identical copies of an original object. The duplicate areas are only parts of the whole images and located in different locations with various of transformations. Thus NDD is a very challenging task, but also a useful practical application.

In this experiment, we combine the INRIA holiday dataset and IPD dataset to construct a large image dataset to conduct the NDD task. To evaluate the performance of different methods in NDD task, we use the images in IPD database as query image. There are 10 image collections which has 200 dupli- 


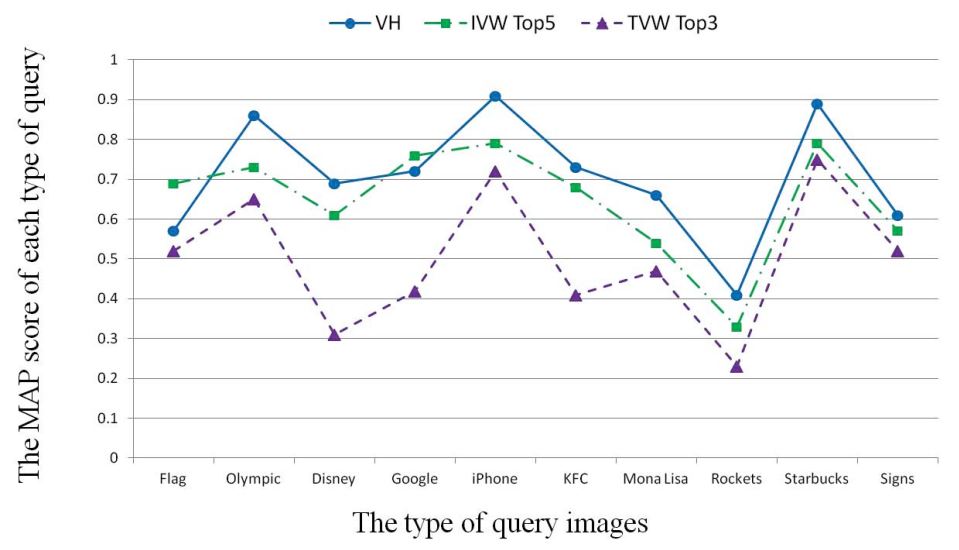

Figure 8: Performance comparison of NDD retrieval with MAP.

cates for each category. Figure 7 illustrates some instances of detection results utilizing our VH method (the lefemost is the query image).

We choose Mean Average Precision (MAP) as the evaluation metric. MAP is calculated for each category with randomly selected query images. Figure 8 demonstrates the comparison between our VH approach with IVW Top5 and TVW Top3. The comparison illustrates the superiority of our VH method to TVW and IVW. However, since we use coarse-partitioning clustering result first to accelerate the training and mapping steps, the MAP performance is unavoidable influenced by the coarse-clustering result. It is a tradeoff between accuracy and speed. With a larger training dataset and a proper expansion method (e.g. considering $\mathrm{n}$ nearest clusters during the training and mapping process), the performance of our VH can be significantly improved.

It is also obvious that even using a totally different training set, our $\mathrm{VH}$ method can still perform well. This demonstrates that the patch similarity definition of $\mathrm{VH}$ is feasible for practical application, and learning local similarity metrics with multiple measures can provide good generalization. 


\subsection{Memory and Time Cost}

305

for practical applications. Learning distribution models is often prohibitively computationally expensive. However, using integrated metrics as section 3 , the generation and assignment of our VH is very efficient.

The similarity propagation process for discovering VHs in the training dataset second.

Although our method needs extra memory to record SVM model for each Visual Homograph, the parameters need to be stored in memory is very limited (only several KB for one model). Therefore the memory cost can be neglected in comparison with traditional visual words.

\section{Conclusion}

In this article, we have explored the appropriate definition of basic visual element to construct more complete and distinctive visual vocabulary, in order to support a wide range of image based applications. Inspired by text retrieval, we have proposed a novel basic visual element called Visual Homograph which denotes groups of patches with indivisible descriptors but extracted from different objects. To generate the Visual Homograph based vocabulary, we have also presented a novel patch similarity metric called Integrated Perceptual Similarity to group relevant patches together. Experimental results on standard datasets demonstrate the superiority of Visual Homograph based method over state-of-the-art visual vocabulary generation approaches, in terms of alleviating incoherence problem, and retrieval performance in practical applications.

Acknowledgements. This work was supported in part by the National Science Foundation of China under Grants No. 61572252, and National Science Foundation of Jiangsu Province under Grants No. BK20150755 and No. BK20150754. 


\section{References}

[1] S. Wei, D. Xu, X. Li, Y. Zhao, Joint optimization toward effective and efficient image search, IEEE Transactions on Cybernetics 43 (6) (2013) 2216-2227.

[2] J. Sivic, A. Zisserman, Video google: A text retrieval approach to object matching in videos, in: IEEE International Conference on Computer Vision, 2003, pp. 1470-1477.

[3] Y. Huang, K. Huang, D. Tao, T. Tan, X. Li, Enhanced biologically inspired model for object recognition, IEEE Transactions on Systems, Man, and Cybernetics, Part B: Cybernetics 41 (6) (2011) 1668-1680.

[4] J. Philbin, O. Chum, M. Isard, J. Sivic, A. Zisserman, Lost in quantization: Improving particular object retrieval in large scale image databases, in: IEEE Conference on Computer Vision and Pattern Recognition, 2008.

[5] Q. Huang, D. Tao, X. Li, L. JIN, G. Wei, Exploiting local coherent patterns for unsupervised feature ranking, IEEE Transactions on Systems, Man, and Cybernetics, Part B: Cybernetics 41 (6) (2011) 1471-1482.

[6] L. Zhang, D. V. Kalashnikov, S. Mehrotra, A unified framework for context assisted face clustering, in: ACM International Conference on Multimedia Retrieval (ACM ICMR 2013), Dallas, Texas, USA, 2013.

[7] L. Zhang, D. V. Kalashnikov, S. Mehrotra, R. Vaisenberg, Context-based person identification framework for smart video surveillance, Machine Vision and Applications (2013) 1-15.

[8] E. Gavves, C. G. Snoek, Landmark image retrieval using visual synonyms, in: ACM International Conference on Multimedia, 2010, pp. 1123-1126.

[9] A. Mikulík, M. Perdoch, O. Chum, J. Matas, Learning a fine vocabulary, in: European Conference on Computer Vision, Springer, 2010. 
[10] F. Moosmann, B. Triggs, F. Jurie, et al., Fast discriminative visual codebooks using randomized clustering forests, in: The Conference on Neural Information Processing Systems, Vol. 2, 2006, p. 4.

[11] T. Zhang, K. Huang, X. Li, J. Yang, D. Tao, Discriminative orthogonal neighborhood-preserving projections for classification, IEEE Transactions on Systems, Man, and Cybernetics, Part B: Cybernetics 40 (1) (2010) 253263.

[12] J. Tang, S. Yan, R. Hong, G.-J. Qi, T.-S. Chua, Inferring semantic concepts from community-contributed images and noisy tags, in: ACM International Conference on Multimedia, 2009, pp. 223-232.

[13] J. Tang, Z.-J. Zha, D. Tao, T.-S. Chua, Semantic-gap-oriented active learning for multilabel image annotation, IEEE Transactions on Image Processing 21 (4) (2012) 2354-2360.

[14] J. C. van Gemert, C. J. Veenman, A. W. M. Smeulders, J. Geusebroek, Visual word ambiguity, IEEE Trans. Pattern Anal. Mach. Intell. 32 (7) (2010) 1271-1283. doi:10.1109/TPAMI.2009.132.

URL http://dx.doi.org/10.1109/TPAMI .2009.132

375 [15] Y. Jiang, C. Ngo, J. Yang, Towards optimal bag-of-features for objec$t$ categorization and semantic video retrieval in: Proceedings of the 6th ACM International Conference on Image and Video Retrieval, CIVR 2007, Amsterdam, The Netherlands, July 9-11, 2007, 2007, pp. 494-501. doi:10.1145/1282280.1282352, URL http: //doi .acm .org/10.1145/1282280.1282352

[16] J. Yang, Y. Jiang, A. G. Hauptmann, C. Ngo, Evaluating bag-of-visualwords representations in scene classification, in: Proceedings of the 9th ACM SIGMM International Workshop on Multimedia Information Retrieval, MIR 2007, Augsburg, Bavaria, Germany, September 24-29, 2007, 2007, pp. 197-206. doi:10.1145/1290082.1290111.

URL http://doi.acm.org/10.1145/1290082.1290111 
[17] Y. Jiang, C. Ngo, Visual word proximity and linguistics for semantic video indexing and near-duplicate retrieval, Computer Vision and Image Understanding 113 (3) (2009) 405-414. doi:10.1016/j.cviu.2008.10.002. URL http://dx.doi.org/10.1016/j.cviu.2008.10.002

[18] Y. Jiang, J. Yang, C. Ngo, A. G. Hauptmann, Representations of keypointbased semantic concept detection: A comprehensive study, IEEE Trans. Multimedia 12 (1) (2010) 42-53. doi:10.1109/TMM.2009.2036235 URL http://dx.doi.org/10.1109/TMM.2009.2036235

[19] K. Mikolajczyk, J. Matas, Improving descriptors for fast tree matching by optimal linear projection, in: IEEE 11th International Conference on Computer Vision, 2007.

[20] J.-M. Morel, G. Yu, Asift: A new framework for fully affine invariant image comparison, SIAM Journal on Imaging Sciences 2 (2) (2009) 438-469.

[21] K. Gao, Y. Zhang, W. Zhang, S. Lin, Affine stable characteristic based sample expansion for object detection, in: ACM International Conference on Image and Video Retrieval, 2010, pp. 422-429.

[22] R.-E. Fan, P.-H. Chen, C.-J. Lin, Working set selection using second order information for training support vector machines, The Journal of Machine Learning Research 6 (2005) 1889-1918.

[23] Z. Wu, Q. Xu, S. Jiang, Q. Huang, P. Cui, L. Li, Adding affine invariant geometric constraint for partial-duplicate image retrieval, in: International Conference on Pattern Recognition, 2010, pp. 842-845.

[24] K. Mikolajczyk, T. Tuytelaars, C. Schmid, A. Zisserman, J. Matas, F. Schaffalitzky, T. Kadir, L. Van Gool, A comparison of affine region detectors, International Journal of Computer Vision 65 (1-2) (2005) 43-72. 